\title{
Der Einfluss der Fügetechnik auf die Konstruktion von Produkten für das Kreislaufwirtschaftssystem
}

\author{
Phillip Wallat ${ }^{1 *}$, Armin Lohrengel ${ }^{1}$ \\ 1 Technische Universität Clausthal, Institut für Maschinenwesen \\ * Korrespondierender Autor: \\ Phillip Wallat, M. Sc. \\ Institut für Maschinenwesen / Institute of Mechanical Engineering \\ Robert-Koch-Straße 32 \\ $D$-38678 Clausthal-Zellerfeld \\ Telefon: +49 5323 / 72-35 07 \\ Mail:wallat@imw.tu-clausthal.de
}

\begin{abstract}
To transform the current economic system towards a circular economy it is necessary to design products accordingly. Many information regarding the suitability of those products are already in existence. Structuring those information and embedding them into the engineering design process will aid the transformation towards a circular economy.

The compatibility of materials during the recycling process is crucial for an efficient recycling, therefor the non-compatible materials have to be separated manually beforehand. Joint technology is the key to be able to do so. Depending on the circular approach the joint technologies differ in their suitability. By link all those information, the engineer can be provided with a tool to directly show the effects of his decisions for the product.
\end{abstract}

\section{Keywords}

Circular Economy, Joint Technology, Engineering Design, Materials, Recycling Technology 


\section{Motivation}

Die Kreislaufwirtschaft stellt einen möglichen Ansatz zur Transformation des aktuellen Wirtschaftssystems dar. Eine recyclinggerechte Konstruktion ist ein Thema, welches bereits seit längerem in der wissenschaftlichen Literatur Beachtung findet. Nicht nur in der VDI 2243 [1] von 2002 wurde das Thema aufgegriffen, auch in neuster Literatur wie BENDER [2] findet es nach wie vor Beachtung. Für die Entwicklung von innovativen Hochtechnologieprodukten sind unter Anderem Rohstoffe notwendig, auf die Deutschland und die EU keinen direkten Zugriff haben. Bereits vor der COVID-19 Pandemie hat die EU eine Liste mit kritischen Rohstoffen und Abhängigkeiten herausgegeben, diese wird weiterhin aktualisiert (vgl. [3]) und stellt damit die Relevanz des Themas dar; die durch die Pandemie noch einmal deutlicher ersichtlich geworden ist.

Eine nachhaltige Konstruktion von Produkten und die zugehörige Recyclingtechnik können ihren Teil dazu beitragen, die vielschichtigen Konsequenzen aus der Abwesenheit / Knappheit bestimmter Rohstoffe zu mindern. Um dies in einem wirtschaftlichen Rahmen durchführen zu können, müssen in der Konstruktion die Faktoren und Restriktionen der Recyclingprozesse so früh wie möglich berücksichtigt werden.

Notwenige Informationen, zur Berücksichtigung vieler Faktoren, sind bereits in großem Umfang vorhanden. Diese durch einen Kontext im Konstruktionsprozess in kurzfristig bereitstellbares Wissen zu transformieren, ermöglicht es, Produkte in kürzerer Zeit auf die Kreislaufwirtschaft anzupassen.

\section{Grundlegende Zusammenhänge von Konstruktionsentscheidung auf Produkte für die Kreislaufwirtschaft}

Entscheidungen innerhalb des Konstruktionsprozesses determinieren die Komplexität des Recyclingprozesses, weiterhin werden durch diese Entscheidungen auch andere Kreislaufansätze erleichtert oder wiederum erschwert (vgl. [1, 2, 4]). In diesem Artikel geht es insbesondere um die Fügetechnik nach DIN 8593 [5] in Abhängigkeit der verwendeten Materialien, unter Berücksichtigung potentieller Kreislaufwirtschaftsansätze; hier Reuse, Remanufacturing, Repair und Recycling. (vgl. [6, 7])

Innerhalb des methodischen Entwicklungsprozesses für Produkte (bspw. nach [2, 8]) werden zur Funktionserfüllung Bauteile entworfen, die durch entsprechende Fügetechnik [5] zu Baugruppen und später zum gesamten Produkt gefügt. Zur übersichtlichen Strukturierung der Zusammenhänge kann die Metus-Raute [2] genutzt werden. Eine verkürzte Version zur Darstellung wesentlicher Zusammenhänge ist in Bild 1 dargestellt. Innerhalb der Produktentwicklung hat vor allem die Funktion bezüglich der gestellten Anforderungen des Produktes häufig Vorrang gegenüber der Recyclingfähigkeit. Daher muss im Grenzfall die Recyclingfähigkeit angepasst werden, dazu sind Detailinformationen zu den Alternativen notwendig.

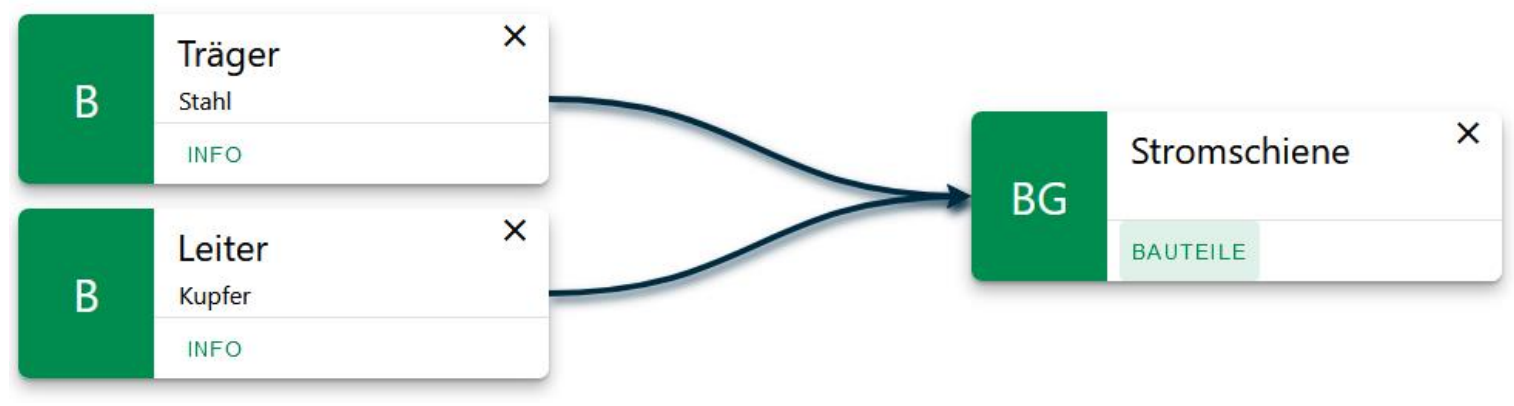

Bild 1: Mögliche Darstellung der Produktarchitektur; in Anlehnung an die Metus-Raute. 
Die Wahl der Fügetechnik beeinflusst maßgeblich wie, sinnvoll ein Kreislaufansatz Anwendung findet und welcher Aufwand abschließend beim Recycling betreiben werden muss, um die im Produkt enthaltenen Materialien und Rohstoffe wieder zu extrahieren. Nach MARTENS [4] stehen innerhalb des Recyclingprozesses Sortierverfahren zur Verfügung. Abhängig von physikalischen Eigenschaften weisen diese Verfahren Sortierpotentiale für Werkstoffe auf; jedoch können nicht alle Materialien beliebig voneinander getrennt und sortiert werden. Dem Sortieren vorgeschaltet sind Klassierungsverfahren zur Korngrößenhomogenisierung der Stoffströme und Verfahren zum Aufschluss der Fügeverbindungen. Diese sind zumeist automatisiert und nicht spezifisch auf ein Produkt oder eine Produktart ausgelegt. Daraus ergibt sich die Notwendigkeit, eine Vielzahl von einzelnen universal Anlagen, zu einem gesamten Recyclingprozess verschaltet, welcher den entsprechenden Input automatisiert zu Sekundärrohstoffen verarbeiten kann.

Durch die Vorbereitung des Inputs in den Gesamtprozess lässt sich der Prozess verkürzen und die Wirtschaftlichkeit erhöhen. Diese Vorbereitung wird bis heute hauptsächlich mittels manuellen Vordemontage durchgeführt. Hierbei sind vor allem der Mechanisierungs-/ und Zerstörungsgrad innerhalb des Demontageprozesses von maßgeblichen Interesse (vgl. [9]).

\section{Vorgehen zur systematischen Lösungsfindung}

Innerhalb des Abschnittes werden die Schritte zum Aufbau eines Informationssystems erläutert, dieses System soll den Konstrukteur bei der methodischen Produktenwicklung von kreislaufgerechten Produkten unterstützen soll. In Bild 2 sind die grundsätzlichen Zusammenhänge des Vorgehens des Abschnitts dargestellt.

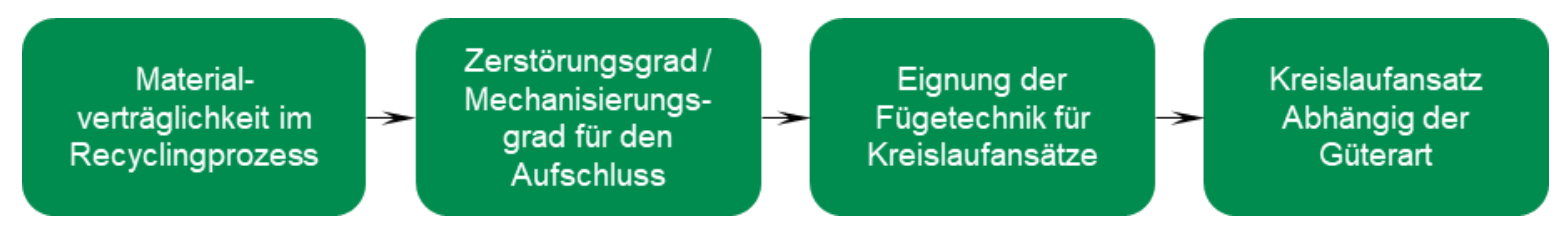

Bild 2: Flussbild zur Erarbeitung der Zusammenhänge

\subsection{Ansatz zur Informationsbereitstellung zur Kreislaufwirtschaft für den Konstrukteur}

Zur Überführung der konstruktiven Entscheidungen in die Eignung für die Kreislaufwirtschaft wurde eine Datenbank erstellt [10], siehe Bild 3. Detaillierte Information zur Erstellung und Ausarbeitung der Datenbank, können [11] entnommen werden. Die Datenbank wurde auf Grundlage des Periodensystems der chemischen Elemente aufgebaut. Für eine Vielzahl chemischer Elemente existieren sowohl Recycling-/ als auch Lösungen für Sortierverfahren. Bekanntlich lassen sich aus den chemischen Elementen Werkstoffe, insbesondere für die Konstruktion, herstellen [12]. Für viele dieser Werkstoffe ist jedoch ein direktes Recycling ohne Separierung aller enthaltener Elemente möglich, solange die geforderte Qualität des Sekundärrohstoffes gegeben ist (vgl. [4]). In diesem Rahmen gibt es zulässige Höchstwerte für einzelne enthaltene chemische Elemente; eine Anreicherung ist daher zu vermeiden. Weiterhin gibt es Störstoffe, welche ein Recycling der Elemente oder Werkstoffe deutlich erschweren.

Der Input in den automatisierten Teil des Recyclingprozesses muss daher frei von Störstoffen und möglichst sortenrein sein, damit ein Recycling wirtschaftlich darstellbar ist. Dies lässt sich über die Fügetechnik realisieren. Dazu muss die angewandte Technik zum Fügen der Bauteile, der Module und des Produktes so ausgelegt werden, dass unerwünschte Werkstoffkombinationen im Vorfeld zuverlässig und wirtschaftlich getrennt werden können. 


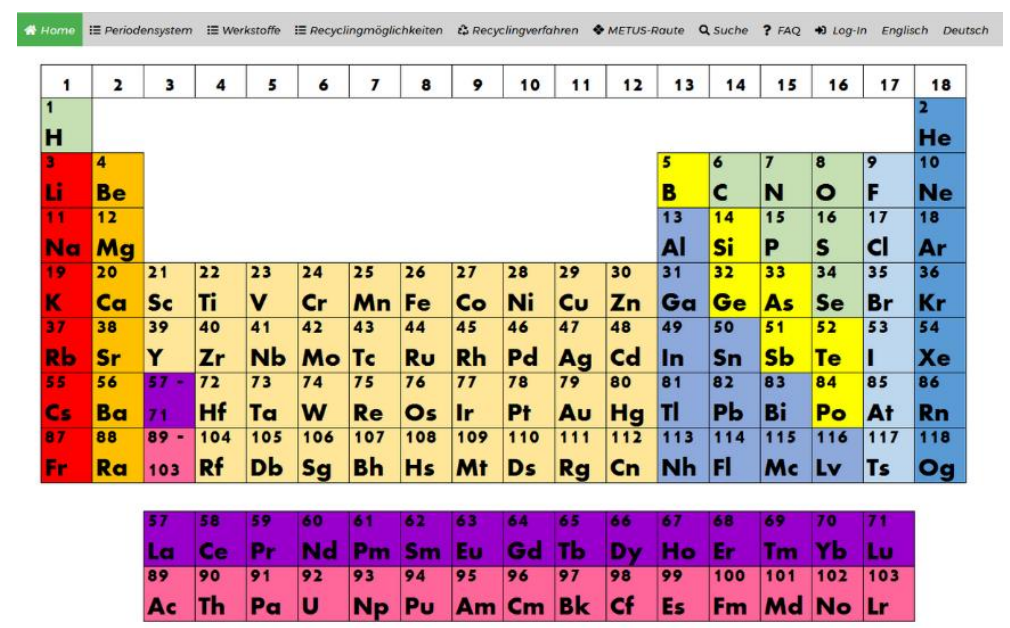

Bild 3: Darstellung der Datenbank, Hauptseite auf Basis der chemischen Elemente

\section{Beispiel:}

Nach MARTENS [4, 12] ist Kupfer mit Stahlwerkstoffen unverträglich, wird zum Fügen beider Fügepartner etwa ein Verfahren aus der Gruppe 4.4, Fügen durch Urformen, nach DIN 8593 [5] gewählt, so ist eine manuelle zerstörungsfreie Vordemontage ohne Werkzeug nicht möglich. Eine zerstörende Demontage mittels einem händisch oder stationär geführtem Werkzeug wäre möglich [9], allerdings nur unter höheren Kosten und Aufwand, die bei höherem Input tendenziell nicht sinken. Daher ist ein mechanisierter / automatisierter Aufschluss wirtschaftlich sinnvoll, allerdings ist ein hoher Aufschlussgrad, durch den hier angenommenen, reinen Formschluss, nur bei entsprechend geringer Korngröße realisierbar. Durch diese geringe Korngrößenverteilung gestaltet sich die anschließende Sortierung nach der Dichte, durch den geringen Dichteunterschied von ca. $1 \mathrm{~g} / \mathrm{cm}^{3}$ (Stahl: 7,8 - 7,9 g/ $\mathrm{cm}^{3}$; Kupfer: $\left.8,9 \mathrm{~g} / \mathrm{cm}^{3}\right)$, als anspruchsvoll und erfordert eine hohe Trennschärfe. Abhängig von der Art des Stahls ist eine magnetische Trennung möglich. Weiterhin ist eine Trennung durch Wirbelstromscheiden anwendbar. Hier ist der Unterschied der Leitfähigkeit zur Dichte mit ca. $6 \mathrm{~m}^{2} \cdot 10^{3} / \Omega \cdot \mathrm{kg}$ (Eisen / Stahl: $1-1,3 \mathrm{~m}^{2} \cdot 10^{3} / \Omega \cdot \mathrm{kg} ;$ Kupfer: $7 \mathrm{~m}^{2} \cdot 10^{3} / \Omega \cdot \mathrm{kg}$ ) relativ hoch (vgl. [4]). Dieser höher dimensionierter Aufwand kann durch die vorherige Auswahl einer funktionell möglichen Fügetechnik verringert werden.

Um diese Entscheidung sinnvoll innerhalb des Produktentwicklungsprozesses einzubetten, ist die Aufbereitung von zahlreichen Informationen notwendig.

- Welche Werkstoffe sind untereinander verträglich / unverträglich?

- Welche Werkstoffkombination mit welcher Fügetechnik sorgt für welchen Aufwand für den jeweiligen Recyclingprozess?

- Welche Fügetechniken sind für welchen Kreislaufansatz sinnvoll?

- Welcher Kreislaufansatz ist für welche Güterart sinnvoll?

\subsection{Materialverträglichkeit im Recyclingprozess und der Einfluss der Fügetechnik}

In Tabelle 1 ist ein Auszug aus den Werkstoffverträglichkeiten innerhalb von Recyclingprozessen aus MARTENS [4] aufgestellt. Der Auszug dient beispielhaft dafür, welche Werkstoffe im Idealfall vor dem automatisierten Recyclingprozess demontiert werden müssen. In Verbindung mit der in 3.1 dargestellten Datenbank von EBERLE [10] lassen sich so informationstechnische Zusammenhänge ableiten, welche dem Konstrukteur leicht zugänglich bereitgestellt werden können. 
Mit weiterem Bezug auf die üblichen Konstruktionswerkstoffe nach MOELLER [12] können weitere Einträge für Werkstoffe, auf Basis der chemischen Elemente, in die Datenbank eingepflegt werden (vgl. 3.1) - wenn notwendig sogar, mit prozentualen Anteilen innerhalb der Werkstoffe und zulässigen Anteilen innerhalb des Recyclingprozesses.

Tabelle 1: Auszug der Werkstoffverträglichkeiten innerhalb von Recyclingprozessen, in Anlehnung an [4]

\begin{tabular}{|c|c|c|c|c|}
\hline Werkstoff & $\begin{array}{l}\text { Hochlegierter } \\
\text { Stahl }\end{array}$ & $\begin{array}{l}\text { Niedriglegierter } \\
\text { Stahl }\end{array}$ & Kupfer & Magnesium \\
\hline verträglich & Mg, Al, Ti, Si & $\mathrm{Mg}, \mathrm{Al}, \mathrm{Ti}, \mathrm{Si}$ & $\begin{array}{l}\mathrm{Ag}, \mathrm{Au}, \mathrm{Hg}, \mathrm{Pd}, \mathrm{Pt} \\
\mathrm{Mg}, \mathrm{Al}, \mathrm{Ti}, \mathrm{Fe}\end{array}$ & keine \\
\hline möglich & $\begin{array}{l}\mathrm{Cr}, \mathrm{Ni}, \mathrm{Mo}, \mathrm{Mn}, \mathrm{S}, \\
\mathrm{Cl}\end{array}$ & $\mathrm{S}, \mathrm{Cl}$ & $\begin{array}{l}\mathrm{Zn}, \mathrm{Sn}, \mathrm{Ni}, \mathrm{Se}, \mathrm{Te} \\
\mathrm{Pb}, \mathrm{Ni}, \mathrm{Sb}, \mathrm{Br}, \mathrm{Cl}\end{array}$ & $\mathrm{Li}$ \\
\hline unverträglich & $\mathrm{Cu}, \mathrm{Sn}, \mathrm{P}$ & $\mathrm{Ni}, \mathrm{Cu}, \mathrm{Mo}, \mathrm{Sn}, \mathrm{P}$ & $\begin{array}{l}\mathrm{Sb}, \mathrm{F}, \mathrm{Cl}, \mathrm{Br} \text {, (hohe } \\
\text { Konzentration) }\end{array}$ & alle \\
\hline
\end{tabular}

Zusammenhänge nach Kanzler [13] zur Fügetechnik nach DIN 8593 [5] sind in Tabelle 2 aufgelistet. Diese Zusammenhänge resultieren aus weiteren detaillierten Untersuchungen zur Fügetechnik und sind durch Bewertungen von einzelnen Faktoren entstanden. Diese werden im Verlauf (siehe Tabelle 5) kurz erläutert. Daraus lassen sich Details zum potentiellen Zerstörungs- und Mechanisierungsgrad nach VDI 2343 [9] ableiten. Dies ist maßgeblich für die Betrachtung der möglichen Vordemontage und damit der Entlastung des automatisierten Recyclingprozesses, wie in Abschnitt 2 beschrieben. Je unverträglicher die Werkstoffe untereinander sind desto, geringer sollte der, möglichst hoch mechanisierte, Zerstörungsgrad innerhalb des Aufschlusses sein.

Tabelle 2: Potentielle Zerstörungs-/ und Mechanisierungsgrade abhängig von der Gruppe der Fügetechnik

\begin{tabular}{|c|c|c|c|c|c|c|}
\hline \multirow[b]{2}{*}{ Fügeverfahren } & \multicolumn{3}{|c|}{ Potentielle Zerstörungsgrade } & \multicolumn{3}{|c|}{$\begin{array}{c}\text { potentieller } \\
\text { Mechanisierungsgrad }\end{array}$} \\
\hline & $\begin{array}{l}\text { zerstörungs- } \\
\text { frei }\end{array}$ & teilzerstörend & zerstörend & gering & mittel & \\
\hline 4.1 Zusammensetzen & $\mathbf{x}$ & & & & $\mathbf{x}$ & $\mathbf{x}$ \\
\hline 4.2. Füllen & $\mathbf{x}$ & & $\mathbf{x}$ & & & $\mathbf{x}$ \\
\hline 4.3 An- und Einpressen & $\mathbf{x}$ & $\mathbf{x}$ & & $\mathbf{x}$ & $\mathbf{x}$ & $\mathbf{x}$ \\
\hline 4.4 Fügen durch Urformen & & $\mathbf{x}$ & $\mathbf{x}$ & $\mathbf{x}$ & $\mathbf{x}$ & $\mathbf{x}$ \\
\hline 4.5 Fügen durch Umformen & $\mathbf{x}$ & $\mathbf{x}$ & $\mathbf{x}$ & $\mathbf{x}$ & $\mathbf{x}$ & $\mathbf{x}$ \\
\hline 4.6 Fügen durch Schweißen & & & $\mathbf{x}$ & & $\mathbf{x}$ & \\
\hline 4.7 Verbinden durch Löten & & $\mathbf{x}$ & $\mathbf{x}$ & & $\mathbf{x}$ & \\
\hline 4.8 Kleben & & $\mathbf{x}$ & & $\mathbf{x}$ & & \\
\hline
\end{tabular}

\subsection{Fügetechnik und deren Eignung für Ansätze der Kreislaufwirtschaft}

Neben der Berücksichtigung des Recyclingansatzes wurden, in der Arbeit von Kanzler [13], die Fügetechniken zusätzlich für weitere produktlebenszeitverlängernden Kreislaufansätze strukturiert.

Aus dieser Aufstellung kann der Konstrukteur alternative Fügetechniken, abhängig vom Kreislaufansatz wählen. Dies ermöglicht es, eine technisch und funktionell vertretbare Fügetechnik zu wählen, wobei diese nicht zwingen die optimale sein muss / kann, wenn dies nicht möglich 
ist. Im Gegenzug ist es ebenfalls möglich, eine zyklophobe ${ }^{1}$ Fügetechniken, mit einem negativen Einfluss für den entsprechenden Kreislaufansatz, zu überdenken und stattdessen eine zyklophile $^{2}$ Fügetechnik zu nutzen. Die Eignung der Fügetechniken ist abhängig vom Kreislaufansatz. Falls eine Fügetechnik für einen bestimmten Ansatz weniger geeignet, allerdings technisch notwendig ist, ist eine Neuausrichtung des Kreislaufansatzes unter Umständen sinnvoll und kann dem Konstrukteur als Alternative aufgezeigt werden.

Tabelle 3: Rangreihung der Fügetechnikeignung für ausgewählte Kreislaufansätze

\begin{tabular}{|c|c|c|c|c|}
\hline $\begin{array}{l}\text { Verfahren und } \\
\text { Ordnungszahlen aus } \\
\text { DIN } 8593\end{array}$ & $\begin{array}{l}0 \\
\mathscr{D} \\
\Phi \\
\simeq \\
\simeq\end{array}$ & 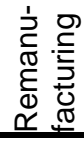 & 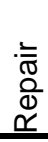 & 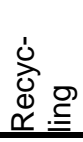 \\
\hline 4.1 Zusammensetzen & 2 & 1 & 1 & 1 \\
\hline 4.2 Füllen & 6 & 5 & 6 & 6 \\
\hline 4.3 An- und Einpressen & 4 & 2 & 3 & 2 \\
\hline 4.4 Urformen & 7 & 8 & 8 & 8 \\
\hline 4.5 Umformen & 8 & 7 & 7 & 3 \\
\hline 4.6 Schweißen & 1 & 4 & 2 & 5 \\
\hline 4.7 Löten & 3 & 3 & 4 & 4 \\
\hline 4.8 Kleben & 5 & 6 & 5 & 7 \\
\hline
\end{tabular}

\subsection{Güterarten, Kreislaufansätze und die Eignung der Fügetechnik.}

Der methodische Ansatz zur Produktentwicklung findet für eine Vielzahl von Güterarten Anwendung. Abhängig von der Art des zu entwickelnden Produktes sind die Kreislaufansätze unterschiedlich zu präferieren.

Tabelle 4: Eignung von Kreislaufansätzen abhängig von der Güterart in Verbindung mit Fügetechniken

\begin{tabular}{|c|c|c|c|c|c|}
\hline \multirow{2}{*}{ Güterart } & \multicolumn{2}{|c|}{ Ansatz } & \multicolumn{3}{|c|}{ Fügetechnik } \\
\hline & Wahl & Name & 1. Wahl & 2. Wahl & 3. Wahl \\
\hline \multirow{3}{*}{ Anlagegut } & 1. & Reman & Zusammensetzen & Ein- / Anpressen & Löten \\
\hline & 2. & Repair & Zusammensetzen & Schweißen & Ein- / Anpressen \\
\hline & 3. & Reuse & Schweißen & Zusammensetzen & Löten \\
\hline \multirow{3}{*}{ Zulieferteile } & 1. & Reuse & Schweißen & Zusammensetzen & Löten \\
\hline & 2. & Repair & Zusammensetzen & Schweißen & Ein- / Anpressen \\
\hline & 3. & Recycling & Zusammensetzen & Ein- / Anpressen & Umformen \\
\hline \multirow{3}{*}{ Sachgüter } & 1. & Repair & Zusammensetzen & Schweißen & Ein- / Anpressen \\
\hline & 2. & Recycling & Zusammensetzen & Ein- / Anpressen & Umformen \\
\hline & 3. & Reuse & Schweißen & Zusammensetzen & Löten \\
\hline \multirow{3}{*}{$\begin{array}{l}\text { Güter des } \\
\text { täglichen } \\
\text { Bedarf }\end{array}$} & 1. & Recycling & Zusammensetzen & Ein- / Anpressen & Umformen \\
\hline & 2. & Reuse & Schweißen & Zusammensetzen & Löten \\
\hline & | 3. & Repair & Zusammensetzen & Schweißen & Ein- / Anpressen \\
\hline
\end{tabular}

\footnotetext{
${ }_{1}^{1}$ Handlung, welche die Kreislaufeignung von Produkten verringert.

${ }^{2}$ Handlung, welche die Kreislaufeignung von Produkten erhöht.
} 
In Tabelle 4 ist eine Auswahl an Güterarten nach KOTLER [14] im Kontext der zu bevorzugenden Kreislaufansätze aufgeführt. Abhängig der jeweiligen Kombination von Art und Ansatz, ist eine gestufte Auswahl von drei Fügetechniken aufgelistet, welche für die entsprechende Kombination als zyklophil eingestuft sind. Diese Auswahlübersicht hilft dem Konstrukteur allerdings vorerst nur bei der vorherigen Abschätzung zu Beginn der Produktentwicklung, um das Gut auf einen Ansatz auszurichten.

Beispiel:

Entwickelt der Konstrukteur ein Sachgut als Produkt, hilft die Übersicht aus Tabelle 4 dabei, sich vorzugsweise für den Kreislaufansatz Repair zu entscheiden. Im Zuge der weiteren Entwicklung kann der Fokus auf die Fügetechniken Zusammensetzen, Schweißen und Ein- / Anpressen mit allen untergliedernden Ordnungsgruppen aus DIN 8593 gelegt werden. Dies geschieht im Idealfall in abhängig von den zu kombinierenden Werkstoffen und deren Verträglichkeit innerhalb des jeweiligen Recyclingprozesses (vgl. Tabelle 1) und dem damit anzustrebenden Zerstörungs- und Demontagegrad (vgl. Tabelle 2).

Wenn dies durch technische und funktionelle Restriktionen mit der Auswahl der Fügetechniken nicht sinnvoll möglich ist, müssen die Fügetechniken zwangsläufig variiert werden. An diesem Punkt steigt die Komplexität für die Berücksichtigung der Kreislaufgerechtigkeit an. Um den Konstrukteur hier zielgerichtet, mit geringen Aufwand zu unterstützten, ist in der Datenbank (vgl. 3.1) ein Interface für zeitnahes Feedback zur jeweiligen Entscheidung angebunden.

\section{IT gestütztes Feedback aus der Datenbank für den Konstrukteur}

Die Ergebnisse aus Abschnitt 3 müssen für eine Anwendung gebündelt und zum entsprechenden Zeitpunkt im Konstruktionsprozess in einem sinnvollen Umfang bereitgestellt werden. Ein reines arbeiten mit den Tabellen - wobei nicht alle, die notwendig wären, innerhalb dieses Artikels enthalten sind - entlastet den Konstrukteur letztendlich nicht; dies würde den Arbeitsaufwand zur Anwendung steigern. Durch die vorherigen stringenten Verknüpfungen der Tabelleninhalte kann sich die Informationstechnologie zu Nutze gemacht werden, um die Anwendung zu erleichtern.

\subsection{Bündelung der Information}

Für ein direktes Feedback innerhalb des Konstruktionsprozesse wird in der Datenbank dazu ein Kreislaufdreieck implementiert, siehe Bild 4. In diesem stellen Lagebereiche für Güterarten und Kreislaufansätze, mit deren Schnittmengen, die Produktausprägungen für ein kreislaufgerechtes Produkt dar. Diese Schnittmenge grenzt so den Entwicklungsbereich, in dem sich der Konstrukteur, unter Berücksichtigung der Güterart und des Kreislaufansatzes, bewegen kann ein. Als Beispielprodukt ist in Rot ein Anlage-Repairprodukt eingezeichnet. Durch die Lage, im Bereich des Repairansatzes und zusätzlich in dem des Anlagengutes hat der Konstrukteur ein Feedback, bei welchem der drei Faktoren noch Spielräume vorhanden sind, um das Produkt in diesem Bereich zu halten. Für weitere Information zur Entwicklung des Kreislaufdreieckes wird die Literatur von [15] empfohlen.

In Tabelle 5 sind in der Kopfzeile die Variablen aus dem Kreislaufdreieck genau aufgeschlüsselt. Bei dem „Wert“ handelt es sich nicht um den rein monetären Wert des Produktes, sondern um die Wertigkeit in Zusammenhang mit der Qualität und den Kosten des Produktes. Die Variable „Gehalt" steht im Bezug zum werkstofflichen Gehalt in Art und Menge. Innerhalb der „Obsoleszenz" sind unterschiedliche Ursachen für die Entstehung dieser berücksichtigt. Nach den Faktoren auf der linken Seite wurden in der Arbeit von KANZLER [13] alle Fügetechniken nach DIN 8593 [5] bewertet. Die Markierungen in der Tabelle stellen die Zusammenhänge quantitativ dar. Damit ist ein informationstechnisch verarbeitbarer Zusammenhang gegeben, was ein direktes Feedback bei Änderung der Fügetechnik für den Konstrukteur über das Kreislaufdreieck zulässt. 


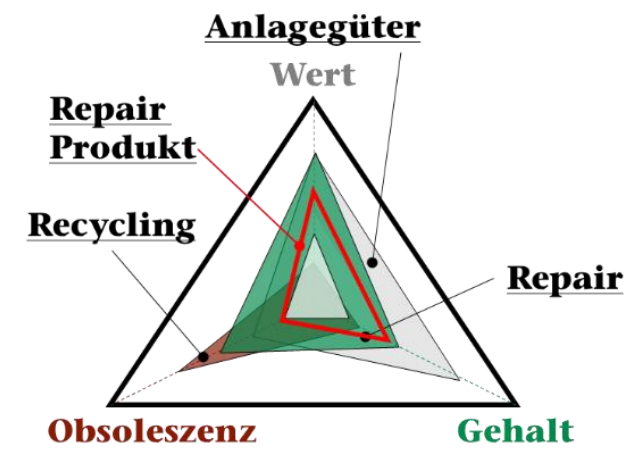

Bild 4: Kreislaufdreieck mit Lagebereichen für Anlagegüter, Recycling und Repair, zentral (in Rot) ist ein aktuelle Ausprägung für ein Repairprodukt mit den aktuellen Ausprägungen eingezeichnet.

Wann und wie der Konstrukteur innerhalb des Konstruktionsprozesses - neben der Entscheidung über die Fügetechnik - Möglichkeiten zur Beeinflussung der Variablen im Kreislaufdreieck - und damit auf die Eignung des Produktes für den jeweiligen Kreislaufansatz - hat, ist in [16] beschrieben.

Tabelle 5: Zusammenhänge und Einflüsse der Fügetechniken auf die Ausprägungen des Kreislaufdreiecks

\begin{tabular}{|c|c|c|c|c|c|c|c|c|}
\hline & Wert & \multicolumn{2}{|c|}{ Gehalt } & \multicolumn{4}{|c|}{ Obsoleszenz } \\
\hline & & Qualität Kosten & Art & Menge & $\begin{array}{l}\text { werk- } \\
\text { stofflich }\end{array}$ & $\begin{array}{c}\text { öko- } \\
\text { nomisch }\end{array}$ & $\begin{array}{l}\text { funk- } \\
\text { tionell }\end{array}$ & $\begin{array}{l}\text { psycho- } \\
\text { logisch }\end{array}$ \\
\hline \multirow{3}{*}{ 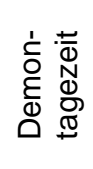 } & Bindungsart & $x$ & $x$ & & & & & \\
\hline & Modularität & $x$ & & & & $x$ & & \\
\hline & Zugänglichkeit & & & & & $x$ & & \\
\hline \multirow{3}{*}{ 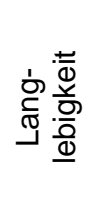 } & Regenerierbarkeit & $x$ & & & & & & \\
\hline & Obsoleszenz & & & & & $x$ & $x$ & $x$ \\
\hline & $\begin{array}{l}\text { Schutz vor Schmutz und } \\
\text { Korrosion }\end{array}$ & $\mathrm{x}$ & & & & & & \\
\hline \multirow{3}{*}{ 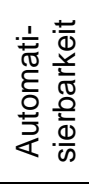 } & Eindeutigkeit & & & & & $x$ & & \\
\hline & Standardisierung & $\mathrm{x}$ & & & & $x$ & & \\
\hline & Lösbarkeit & $x$ & & & & $x$ & & \\
\hline \multirow{4}{*}{$\begin{array}{l}\frac{7}{0} \\
\frac{0}{00} \\
\frac{2}{50} \\
3\end{array}$} & Recyclingeignung & & $x$ & $x$ & $x$ & $x$ & & \\
\hline & Recyclingverträglichkeit & & $x$ & $x$ & $x$ & $x$ & & \\
\hline & Trennung & & $x$ & $x$ & & & & \\
\hline & Leichtbau & $x$ & $x$ & $x$ & & & & \\
\hline
\end{tabular}

\subsection{Weitere geplante Entwicklungsschritte der Inhalte}

Die Inhalte der in diesem Beitrag enthaltenen Tabellen müssen für eine höhere Belastbarkeit noch weiter verifiziert werden. Die Bewertung der Einflussfaktoren der Fügetechnik aus Tabelle 3 und Tabelle 5 hat bereits eine weitere kritische Betrachtung durch ZHANG [17] erhalten. Die daraus resultieren Abstufungen nach ergänzenden Kriterien müssen zukünftig noch mit in die Bewertung einfließen.

Weiterhin gilt es, die Verträglichkeiten der chemischen Elemente, aus informationstechnisch Sicht, in eine saubere Form zu bringen (vgl. Tabelle 1). Diese muss beispielsweise 
berücksichtigen, dass Kupfer als Werkstoff nicht im Stahlwertstoffströmen enthalten sein darf. Eisen, als Hauptbestandteil von Stahl, kann jedoch durchaus im Recyclingprozess von Kupfer enthalten sein.

Für das Kreislaufdreieck müssen die Lagebereiche genauer verifiziert und mit messbaren Werten hinterlegt werden. Die bisher enthaltene Abschätzung liegt zwar in einem nachvollziehbaren Bereich, die Betrachtung im Detail ist dennoch für die weitere Entwicklung zwingend notwendig.

\section{Zusammenfassung und Ausblick}

Die vorgestellte Systematik hat nach weiterer Entwicklung das Potential, den Konstruktionsprozess, speziell für Kreislaufgerechte Produkte, gezielt und auf Basis von belastbaren Größen zu unterstützen. Dafür sind in den einzelnen Schritten noch weitere und detailliertere Informationen notwendig. Allerdings ist in der bisherigen Struktur das zugrundeliegende System bereits als zielführender Ansatz erkennbar.

Es gibt neben den in Abschnitt 2 angesprochenen vier Kreislaufansätze noch weitere weitere Definitionen. Ein System der „9R“ aus POTTING [18] nach [19] stellt weitere Ansätze mit Einflüssen in der Produktion wie Refuse, Rethink und Reduce dar. Weiterhin ist mit Recover ein Kreislaufansatz nach dem Recycling möglich. Eine Auswertung von 114 Ansatzdefinitionen für die Kreislaufwirtschaft wurde in KIRCHHERR [20] durchgeführt. Durch die offene und erweiterbare Bewertungsstruktur ist es möglich, weitere Ansätze in die Systematik zu implementieren.

Ähnliches gilt für weitere Verfahren der Fertigungstechnik nach DIN 8580 [21]. Aus der Norm und der zugehörigen Struktur können weitere Fertigungsverfahren in das System eingebunden werden, welche maßgeblichen Einfluss auf die Kreislaufeignung von Produkten haben. Dabei ist stets abzuwägen, ob die zusätzliche Information für den Konstrukteur noch eine Hilfestellung darstellt oder eine unerwünschte Mehrbelastung ohne entsprechenden Mehrwert erzeugt.

Für das Kreislaufdreieck (vgl. Bild 4) lassen sich weiterhin die bereits vorhandenen Faktoren weiter auffächern und neue Faktoren hinzufügen, welche weitere Einflüsse berücksichtigen. Wird dies zusätzlich mit der Wahlmöglichkeit einer Grob-/ oder Feindarstellung während des Entwicklungsprozesses kombiniert, kann sich der Konstrukteur entweder einen Überblick verschaffen oder gezielt an Details arbeiten um das Produkt für die Kreislaufwirtschaft zu optimieren.

Für das Gesamtkonzept der Kreislaufwirtschaft müssen weitere Stakeholder beachtet werden. Aus dem System lassen sich Informationen ableiten, welche relevant für weitere Akteure sind. Gerade die Produktarchitektur - inklusive der angewandten Fügetechniken - und zusätzlich die bereits hinterlegten Informationen der notwendigen Recyclingprozesse, können bei größeren Stückzahlen von Produkten als Planungsunterstützung für Recyclingbetrieb dienen. In Zusammenhang mit der voraussichtlichen Produktlebensdauer, Anzahl der Produkte und Kenntnis der enthaltenen recycelbaren Rohstoffe lassen sich mögliche Ankunftsraten von Rohstoffen mit einer gewissen Preisstabilität abschätzen. Dies stellt eine Art planbare Sekundärrohstoffquelle dar. Welche zusätzlichen neun Herausforderungen sich aus der Weitergabe dieser Informationen an weitere Stakeholder ergeben sind in [22] andiskutiert. 


\section{Literaturverzeichnis}

[1] VDI-Richtlinie 2243:2002-07. VDI 2243 - Recyclingorientierte Produktentwicklung

[2] Bender, B. u. Gericke, K.: Pahl/Beitz Konstruktionslehre. Methoden und Anwendung erfolgreicher Produktentwicklung. Berlin, Heidelberg: Springer Berlin Heidelberg; Imprint: Springer Vieweg 2021

[3] Widerstandsfähigkeit der EU bei Kritischen Rohstoffen: Einen Pfad hin zu größerer Sicherheit und Nachhaltigkeit abstecken. COM(2020) 474 final, Europäische Kommission, 2020

[4] Martens, H. u. Goldmann, D.: Recyclingtechnik. Fachbuch für Lehre und Praxis. Wiesbaden: Springer Vieweg 2016

[5] DIN Norm 8593:2003-09. DIN 8593 - Fertigungsverfahren Fügen

[6] Goodship, V., Stevels, A. u. Huisman, J.: Waste electrical and electronic equipment (WEEE) handbook. Woodhead publishing series in electronic and optical materials. Duxford: Elsevier, WP, Woodhead Publishing 2019

[7] Langefeld, O. u. Mrotzek-Blöß, A. (Hrsg.): Forschungsfeld Rohstoffsicherung und Ressourceneffizienz. Forschungsfeldkolloquium 2020. Clausthal-Zellerfeld: Papierflieger 2020

[8] VDI 2221 - Entwicklung technischer Produkte und Systeme

[9] VDI-Richtlinie 2343 Blatt 3:2009-04. VDI 2343 - Recycling elektrischer und elektronischer Geräte Demontage

[10] Christof Eberle: Erstellung einer Datenbank zu Recyclingverfahren für die Elemente des Periodensystems, Technische Universität Clausthal Masterarbeit. Clausthal-Zellerfeld 2020

[11] Phillip Wallat u. Armin Lohrengel: Erstellung einer Materialdatenbank zur digitalen Systematisierung im Konstruktionsprozess für kreislaufgerechte Produkte. 4. Symposium Materialtechnik. Band 10. 2021, S. 526536

[12] Moeller, E. (Hrsg.): Handbuch Konstruktionswerkstoffe. Auswahl, Eigenschaften, Anwendung. München: Hanser 2014

[13] Edward Kanzler: Analyse und Bewertung der Fügeverfahren nach DIN 8593 und deren Einflusses auf eine kreislaufgerechte Konstruktion, Technische Universität Clausthal Masterarbeit. Clausthal-Zellerfeld 2021

[14] Kotler, P., Armstrong, G., Harris, L. C. u. Piercy, N. F.: Grundlagen des Marketing. Hallbergmoos: Pearson 2019

[15] Phillip Wallat u. Armin Lohrengel: Wirtschaftliche Einflussfaktoren auf eine kreislaufgerechte Produktentwicklung. In: Langefeld, O. u. Mrotzek-Blöß, A. (Hrsg.): Forschungsfeld Rohstoffsicherung und Ressourceneffizienz. Forschungsfeldkolloquium 2020. Clausthal-Zellerfeld: Papierflieger 2020, S. 69-79

[16] Phillip Wallat u. Armin Lohrengel: Die Einbettung kreislaufgerechter Konstruktionsansätze in den Produktentstehungsprozess. In: 18. Gemeinsames Kolloquium Konstruktionstechnik 2020: Nachhaltige Produktentwicklung: KT 2020. DuEPublico: Duisburg-Essen Publications online, University of DuisburgEssen, Germany 2020, S. 163-174

[17] Xiaoran Zhang: Systematische Beschreibung und Bewertung der Fügeverfahren nach DIN 8593, auf Gruppen-/ und Untergruppen-Ebene bezüglich vier unterschiedlicher Ansätze der Kreislaufwirtschaft, Technische Universität Clausthal Bachelorarbeit. Clausthal-Zellerfeld 2021

[18] Potting, J., Hekkert, M., Worrell, E. u. Hanemaaijer, A.: Circular Economy: Measuring Innovation in the Product Chain. Policy Report. The Hague 2017

[19] Gudde, C. (Hrsg.): Circulaire economie: van wens naar uitvoering. Den Haag: Raad voor de leefomgeving en infrastructuur 2015

[20] Kirchherr, J., Reike, D. u. Hekkert, M.: Conceptualizing the circular economy: An analysis of 114 definitions. Resources, Conservation and Recycling 127 (2017) 3, S. 221-232

[21] DIN Norm 8580:2020-01. DIN 8580 - Fertigungsverfahren. Begriffe, Einteilungen

[22] Lawrenz, S., Nippraschk, M., Wallat, P., Rausch, A., Goldmann, D. u. Lohrengel, A.: Is it all about Information? The Role of the Information Gap between Stakeholders in the Context of the Circular Economy. Procedia CIRP 98 (2021), S. 364-369 